\title{
DESARROLLO PONDERAL DE TERNEROS BUBALINOS (Bubalus bubalis) CRIADOS SIN AMAMANTAMIENTO NATURAL
}

Elena Katayarna Cruz

$M$ anuel Isujza Cabrera ${ }^{* *}$

\section{RESUMEN}

EI experimento se realizó en el Centro de Investigaciones "Jenaro Herrera" del Instituto de Investigaciones de la A mazonía Peruana, IIAP, que se encuentra ubicado en el distrito de J enaro Herrera a $150 \mathrm{~K} \mathrm{~m}$. de la ciudad de Iquitos, en la margen derecha del río U cayali a 120 msnm aproximadamente. En el trabajo se planteó determinar la dosis adecuada de leche para la alimentación de terneros bubalinos criados sin amamantamiento natural mediante evaluaciones del desarrollo ponderal de éstos, para ello, se estudiaron tres niveles de alimentación láctea 10, 12 y 14 por ciento del peso vivo, hasta un período de lactancia de doce semanas. Los terneros al nacer fueron separados definitivamente de sus madres y alimentados con leche en cantidades que variaron con la edad, peso y las características de las dosis experimentales. Se midieron los pesos al nacer y pesos semanales durante todo el experimento, así como la leche consumida; para el análisis estadístico se utilizó el DCA y los análisis de regresión y correlación lineal. Los resultados muestran que los incrementos de peso vivo obtenidos al destete fueron $4.40 ; 5.75$ y $6.21 \mathrm{~kg} / \mathrm{sem} / \mathrm{ter}$, en los tratamientos $\mathrm{A}, \mathrm{B}$, y C, respectivamente; existiendo una asociación significativa y consistente entre el incremento de peso frente al consumo de leche. Respecto a este consumo, la mejor conversión alimenticia láctea se observa en el tratamiento B (5.76).

\section{INTRODUCCION}

La crianza del búfalo de agua (Bubalus bubalis) resulta una alternativa para mejorar la producción pecuaria en la región amazónica; así lo indican los satisfactorios resultados obtenidos por la Universidad Nacional de la A mazonía Peruana, el Centro de Investigaciones Jenaro Herrera y el Proyecto SorgoBúfalos en Iquitos. A semejanza de otras especies de animales domésticos, la crianza de los búfalos puede efectuarse utilizando los sistemas: extensivo, semintensivo e intensivo; sin embargo, su rusticidad y capacidad de producción en leche, carne y tracción en base a una alimentación con pastos y forrajes de

\footnotetext{
* Ingeniero A grónorno. Investigador del CIJH-IIAP

** Ingeniero A grónomo. Director del $\mathrm{CIJH}$ - IIAP
} 
baja calidad, constituyen urna ventaja que le permite desenvolverse en condiciones de la A mazonía, EM BRA PA (1981).

En el Centro de Investigaciones Jenaro Herrera a los búfalos de agua se los cría en forma semintensiva y en ellos se realizan estudios de alimentación para la producción de leche y carne en base a raciones económicas. Específicamente, en la crianza de los terneros hay necesidad de definir un programa de alimentación que asegure el crecimiento vigoroso de los animales y permita el mínimo consumo de leche durante el período de lactancia; mejor aún, si se lograra disminuir el 'stress' posterior al destete. En tal sentido, los objetivos del presente estudio son: determinar la dosis adecuada de leche en la alimentación de terneros bubalinos criados sin amamantamiento natural, y evaluar el desarrollo ponderal de éstos.

\section{MATERIALES Y METODOS}

\section{LOCALIZACION DEL EXPERIMENTO Y CLIMA}

El trabajo se realizó en el Centro de Investigaciones "Jenaro Herrera", como parte del proyecto "Sistemas Integrales de Producción en J enaro Herrera" situado en el distrito de Jenaro Herrera de la Región Loreto a $150 \mathrm{~km}$ de la ciudad de Iquitos; el trabajo duró de febrero de 1992 mayo de 1993.

En la zona de estudio las temperaturas medias mensuales oscilan entre 25.2 y $26.90 \mathrm{C}$, con un promedio de 26 50 C humedad relativa de 85 por ciento, y una precipitación media anual de $2687 \mathrm{~mm}$. que varía entre 1700 y 4000 mm., M arengo (1983).

\section{DISTRIBUCION DE LOS ANIMALES Y TRATAMIENTOS}

En el experimento se emplearon doce terneros bubalinos de ambos sexos, con los cuales se formaron tres grupos. Los animales integraban cada grupo a medida que nacían y en forma indistinta; con la finalidad de realizar una azarización adecuada.

Se estudiaron tres niveles de alimentación láctea 10, 12 y 14 por ciento del peso vivo; cada nivel constituyó un tratamiento.

\section{ALIMENTACION Y MANEJO DE LOS TERNEROS}

Después del parto a los terneros se los separó definitivamente de sus madres, procediendo luego) a la desinfección del cordón umbilical con una solución yodada. 
En todos los terneros se midió los pesos al nacer y en lo sucesivo se realizó semanalmente controles de peso, por las mañanas y después de transcurrido doce horas de la última alimentación correspondiente a cada semana. Los siete primeros días, los terneros recibían ad libitum el calostro en dos tomas diarias: mañana y tarde; en este período se enseña a los terneros a tomar la leche en baldes. En el siguiente cuadro se indica la distribución de las raciones de leche y contenido que se va a seguir en los tratamientos.

\section{Cuadro 1}

\section{Programa de Alimentación con leche y concentrado para terneros bubalinos} criados sin amamantamiento natural

\begin{tabular}{|c|c|c|c|c|c|}
\hline $\begin{array}{l}\text { EDAD } \\
\text { (semi) }\end{array}$ & $\begin{array}{l}\text { PESOS } \\
\text { CONCENT } \\
(\mathrm{kg})\end{array}$ & Ttto A. & $\begin{array}{l}\text { Ttto B. } \\
\text { che kg/día) }\end{array}$ & Ttto & $\begin{array}{l}\text { 16\% P.C. } \\
\text { (Kg/día) }\end{array}$ \\
\hline $0-1$ & $\mathrm{X}_{0} *$ & Calostro** & Calostro** & Calostro** & - \\
\hline $1-2$ & $X_{1}$ & $X_{1} \cdot .10 .0 \%$ & $X_{1 .} 12.0 \%$ & $X_{1 .} 14.0 \%$ & - \\
\hline $2-3$ & $X_{2}$ & $X_{2} .10 .0 \%$ & $X_{2 .} 12.0 \%$ & $X_{2} .14 .0 \%$ & - \\
\hline $3-4$ & $X_{3}$ & $X_{3} .10 .0 \%$ & $X_{3} .12 .0 \%$ & $X_{3} .14 .0 \%$ & - \\
\hline $4-5$ & $X_{4}$ & $X_{4} .7 .5 \%$ & $X_{4}, 9.0 \%$ & $X_{4} .10 .5 \%$ & - \\
\hline $5-6$ & $X_{5}$ & $X_{5} .7 .5 \%$ & $X_{5} .9 .0 \%$ & $X_{5} .10 .5 \%$ & - \\
\hline $6-7$ & $X_{6}$ & $X_{6} .5 .0 \%$ & $6.0 \%$ & $X_{6} .7 .0 \%$ & 0.250 \\
\hline $7-8$ & $X_{7}$ & $X_{7} .5 .0 \%$ & $6.0 \%$ & $X_{7} \cdot 7.0 \%$ & 0.250 \\
\hline 8-9 & $X_{8}$ & $X_{8} .5 .0 \%$ & $X_{8} .6 .0 \%$ & $X_{8} .7 .0 \%$ & 0.500 \\
\hline $9-10$ & $X_{9}$ & $X_{9} .5 .0 \%$ & $X_{9} .6 .0 \%$ & $X_{9} .7 .0 \%$ & 0.500 \\
\hline $10-11$ & $X_{10}$ & $X_{10.2 .5 \%}$ & $X_{10 .} 3.0 \%$ & $X_{10 .} 3.5 \%$ & 0.750 \\
\hline $11-12$ & $X_{11}$ & $X_{11.2 .5 \%}$ & $X_{11 .} 3.0 \%$ & $X_{11 .} 3.5 \%$ & 1.000 \\
\hline
\end{tabular}

$(*) \quad$ Peso al nacer

(**) A limentación ofrecida Ad libitum

De acuerdo al Programa de Alimentación también se ofreció a los terneros, un concentrado con 16 por ciento de proteína bruta en cantidades que variaron con la edad, desde la sexta semana hasta el año de edad. A partir de la décima semana se los conduce a las áreas de pasturas y por las tardes descansan en sus corrales hasta el siguiente día. El destete se realizó en forma brusca a las doce semanas de edad. 


\section{MATERIALES Y EQUIPO DE TRABAJO}

Los materiales empleados en el experimento son :

- Balanza portátil A LFASA de 20 kg de capacidad

- Balanza tipo plataforma SU PER de $1000 \mathrm{~kg}$ de capacidad

- Baldes plásticos

- Libretas de campo

Entre los equipos se considera el equipo de manejo (cuerdas de nylon de 1/2" de diámetro, instrumentos de marcación) y el equipo veterinario (termómetro rectal, jeringas y agujas hipodérmicas, desinfectantes, medicinas, otros).

\section{MEDIDAS REGISTRADAS}

Se registraron en fichas de nacimiento a los animales nacidos durante los meses de estudio. Así como el peso al nacer y pesos semanales; siendo cada ternero evaluado hasta los siete meses de edad. También se registré el consumo de leche diario durante el tiempo de la lactancia para calcular la conversión alimenticia láctea en cada uno de los tratamientos.

\section{DISPOSICION EXPERIMENTAL}

Se ha empleado el Diseño Completamente A leatorio con tres tratamientos y cuatro repeticiones.

Asimismo, se determiné los cálculos de regresión y correlación lineal entre el incremento de peso del ternero frente a la cantidad de leche consumida, peso al nacer y edad del ternero.

\section{RESULTADOS Y DISCUSION}

En los cuadros 2 y 3 se presenta los incrementos de peso vivo logrados en el período de lactancia y postdestete, acompañados de su respectivo análisis. 


\section{Cuadro 2}

Incremento de peso vivo promedio semanal en el período de lactación ( $\mathrm{kg} /$ ternero)

\begin{tabular}{|c|c|c|c|c|}
\hline \multirow{2}{*}{ REPETICION } & \multicolumn{3}{|c|}{ TRATAMIENTO } & TOTAL \\
\cline { 2 - 4 } & $\mathrm{A}$ & $\mathrm{B}$ & $\mathrm{C}$ & REPETICION \\
\hline 1 & 4.67 & 6.33 & 6.25 & 17.25 \\
2 & 4.00 & 5.42 & 6.75 & 16.17 \\
3 & 4.25 & 5.25 & 7.00 & 16.50 \\
4 & 4.67 & 6.00 & 4.83 & 15.50 \\
\hline TOTALES & 17.59 & 23.00 & 24.83 & 65.42 \\
MEDIAS & 4.40 & 5.75 & 6.21 & 5.45 \\
\hline
\end{tabular}

\section{A N V A}

\begin{tabular}{|l|c|c|c|c|}
\hline F. de V & G.L & S.C & C.M & F.C. \\
\hline TRATAMIENTO & 2 & 7.08 & 3.54 & $8.23 * *$ \\
ERROR & 9 & 3.91 & 0.43 & \\
TOTAL & 11 & 10.99 & & \\
\hline
\end{tabular}

C.V. $=12.03 \%$

PRUEBA DE DUNCAN:

\begin{tabular}{|c|c|c|c|}
\hline No & TTTO & MEDIAS & $\begin{array}{c}\text { SIGNIFICACION } \\
*\end{array}$ \\
\hline 01 & C & 6.21 & $a$ \\
02 & B & 5.75 & a \\
03 & A & 4.40 & b \\
\hline
\end{tabular}

* Promedios unidos por una misma letra no difieren estadísticamente. 
E. Katayama C. y M. Isuiza C.

\section{Cuadro 3}

Incremento de peso promedio semanal en el periodo postdestete (K g/ternero

\begin{tabular}{|c|c|c|c|c|}
\hline \multirow{2}{*}{ REPETICION } & \multicolumn{3}{|c|}{ TRATAMIENTO } & TOTAL \\
\cline { 2 - 4 } & $\mathrm{A}$ & $\mathrm{B}$ & $\mathrm{C}$ & REPETICION \\
\hline 1 & 2.25 & 2.95 & 3.00 & 8.20 \\
2 & 3.40 & 3.45 & 3.90 & 10.75 \\
3 & 3.35 & 3.85 & 3.30 & 10.50 \\
4 & 4.00 & 3.45 & 3.70 & 11.15 \\
\hline TOTALES & 13.00 & 13.70 & 13.90 & 40.60 \\
MEDIAS & 3.25 & 3.43 & 3.50 & 3.38 \\
\hline
\end{tabular}

\section{A N V A}

\begin{tabular}{|l|c|c|c|c|}
\hline F. de V & G.L & S.C & C.M & F.C. \\
\hline TRATAMIENTO & 2 & 0.12 & 0.06 & 0.21 N.S \\
ERROR & 9 & 2.48 & 0.28 & \\
TOTAL & 11 & 2.60 & & \\
\hline
\end{tabular}

NS No significativo

C.V. $=15.66 \%$

Con respecto a la alimentación con leche, en el cuadro 4 se indica las cantidades consumidas por los terneros acompañados de su respectivo análisis de varianza. La conversión alimenticia y el costo de la alimentación con leche obtenida en el experimento se los ofrece en el cuadro 5.

\section{Cuadro 4}

Consumo de leche promedio semanal ( $\mathrm{Kg}$./ternero)

\begin{tabular}{|c|c|c|c|c|}
\hline \multirow{2}{*}{ REPETICION } & \multicolumn{3}{|c|}{ TRATAMIENTO } & TOTAL \\
\cline { 2 - 4 } & $\mathrm{A}$ & $\mathrm{B}$ & $\mathrm{C}$ & REPETICION \\
\hline 1 & 28.76 & 37.83 & 37.98 & 104.57 \\
2 & 26.82 & 31.50 & 45.70 & 104.02 \\
3 & 28.83 & 36.59 & 47.53 & 112.02 \\
4 & 28.76 & 38.69 & 31.75 & 99.20 \\
\hline TOTALES & 113.17 & 144.61 & 162.86 & 420.64 \\
MEDIAS & 28.29 & 36.15 & 40.72 & 35.05 \\
\hline
\end{tabular}




\section{A N V A}

\begin{tabular}{|l|c|c|c|c|}
\hline F. de V & G.L & S.C & C.M & F.C. \\
\hline TRATAM IENTO & 2 & 315.89 & 157.94 & $7.41^{*}$ \\
ERROR & 9 & 191.78 & 21.31 & \\
TOTAL & 11 & 507.67 & & \\
\hline
\end{tabular}

* Significativo

$$
\text { C.V. }=13 \cdot 17 \%
$$

PRUEBA DE DUNCAN:

\begin{tabular}{|c|c|c|c|}
\hline No & TTTO & MEDIAS & $\begin{array}{c}\text { SIGNIFICACION } \\
*\end{array}$ \\
\hline 01 & C & 40.72 & a \\
02 & B & 36.15 & a \\
03 & A & 28.29 & b \\
\hline
\end{tabular}

* Promedio unidos por una misma letra no difieren estadísticamente

En el análisis de regresión y correlación lineal el incremento de peso en el período de lactancia está asociado positivamente con el consumo de leche, el coeficiente de correlación es de $0.949 * *$. La línea de regresión que indica esta estrecha relación se presenta en el gráfico respectivo.

\section{DESARROLLO PONDERAL}

En el cuadro 2 se muestra que los incrementos de pesos alcanzados por los terneros en el período de lactancia fue $4.40 ; 5.75$ y $6.21 \mathrm{Kg}$. $/ \mathrm{sem} / \mathrm{ter}$. en los tratamientos $A, B$ y $C$, respectivamente; resultando un aumento lineal a medida que se elevaba los volúmenes de leche.

AI hacer el análisis de varianza se encontró que las diferencias observadas son altamente significativas; es decir, el consumo de leche (en C, B y A) tuvo distintos efectos en el crecimiento del ternero, en la prueba de Duncan los tratamientos $\mathrm{C}$ y $\mathrm{B}$ no difieren estadísticamente.

De acuerdo a los resultados, el incremento de peso de los terneros está en función de la cantidad de leche consumida; pues a medida que aumenta el consumo de leche también aumenta el incremento de peso. Al comparar con los resultados de López (1989) en A rgentina, que obtuvo ganancias de $0.43 \mathrm{Kg}$./ día ó $3.00 \mathrm{Kg}$./sem al experimentar en terneros Holando A rgentino en una lactancia 
de cinco semanas, los incrementos de peso resultan superiores, al igual que, con las respuestas conseguidas por A hmed y EI Shazly (1978) en Egipto en el destete de terneros bubalinos a los cuatro meses de edad, quienes obtuvieron ganancias de $0.33 \mathrm{Kg}$./día ó $2.31 \mathrm{Kg}$./sem cuando alimentaban en baldes; y cuando otros terneros amamantaban al pie de la madre, $4,41 \mathrm{Kg}$./sem que resulta parecida a las ganancias del tratamiento $A$; sin embargo, inferiores a los de $C$ y $B$.

También son superiores a los resultados de Zambrano, et al (1992), quienes en el Proyecto Sorgo-Búfalos en Iquitos evaluaron el crecimiento de terneros bubalinos criados con búfalos nodrizas, en esta evaluación encontraron ganancias de $0.535 \mathrm{Kg}$./día ó $3.74 \mathrm{Kg}$./sem, al destete.

En el período postdestete, los incrementos de peso (cuadro 3) no difieren estadísticamente; lo que nos indica que la ración de leche suministrada en la etapa de lactancia no ha influido en las ganancias de peso postdestete, ya que se tiene incrementos de peso similares $(A=3.25 ; B=3.43$ y $C=3.50 \mathrm{Kg}$. $/ \mathrm{sem} /$ ter $)$. 


\section{Cuadro 5}

\section{Conversión alimenticia y costo de la alimentación láctea}

\begin{tabular}{|c|c|c|c|c|c|}
\hline TRATAM IENTO & $\begin{array}{l}\text { CONSUMO DE LECHE } \\
\text { TOTAL }(\mathrm{kg})\end{array}$ & $\begin{array}{l}\text { INCREMENTO DE } \\
\text { P.V. TOTAL }(\mathrm{kg})\end{array}$ & $\begin{array}{l}\text { CONVERSION } \\
\text { ALIMENTICIA }\end{array}$ & $\begin{array}{l}\text { COSTO/KG DE } \\
\text { LECHE (S/.) }\end{array}$ & $\begin{array}{l}\text { COSTO POR } \\
\text { IN CREM ENTO } \\
\text { DE P.V. (S/.kg) }\end{array}$ \\
\hline $\begin{array}{ll}\text { A } & \\
& 5.90\end{array}$ & $1,245.00$ & 211 & 5.90 & 1.00 & \\
\hline $\begin{array}{l}\text { B } \quad 5.76\end{array}$ & $1,590.70$ & 276 & 5.76 & 1.00 & \\
\hline C & $1,791.45$ & 298 & 6.01 & 1.00 & 6.01 \\
\hline
\end{tabular}


Sin embargo, al comparar las ganancias de peso obtenidas con las encontradas por López (1989) en terneros Holando A rgentino (0.686 kg/día ó $4.80 \mathrm{~kg} / \mathrm{sem}$ ) y Josifovich (1990) en terneros Hereford ( $0.765 \mathrm{~kg} /$ día ó $5.36 \mathrm{~kg} / \mathrm{sem})$, a los siete meses de edad, nuestras respuestas resultan ser de menores ganancias. A simismo, Zambrano, et al (1992) lograron ganancias de $0.509 \mathrm{~kg} /$ día ó $3.56 \mathrm{~kg} / \mathrm{sem}$ en terneros bubalinos de la misma edad.

Estas elevadas ganancias de peso, pueden deberse a la calidad de animales utilizados (de raza pura o mejorada) por los autores, así como al sistema intensivo de alimentación que emplean. Para efectos del trabajo, los animales estudiados se alimentaron de pastos naturales y recibieron una mezcla alimenticia sólo una vez al día.

\section{CONSUMO DE LECHE}

L a leche suministrada en cada tratamiento (en promedio semanal) se registra en el cuadro 4. Las raciones de leche no han sido aplicadas a plenitud en todos los tratamientos, siendo el consumo de leche normal (lo consumían todo) en los tratamientos B (12\%) y A $(10 \%)$; no dándose así en el tratamiento C (14\%) por presentarse problemas en la capacidad de ingerir toda la leche.

El análisis de varianza nos indica que existe diferencia significativa y al determinarse la prueba de Duncan encontramos que hay diferencias entre el tratamiento $A$ con $C$ y $B$, no así entre $C$ y $B$. Sin embargo, al observar las conversiones alimenticias ( $\mathrm{kg}$ de leche/ $\mathrm{kg}$ de incremento de peso) expuestos en el cuadro 5; el mejor índice de conversión se encontró en el tratamiento B (5.76); además que resultó ser el programa de menor costo.

\section{CONCLUSIONES}

a) Los incrementos de pesos en el período de lactancia fueron lineales; es decir en relación directa al aumento de leche.

b) El consumo de leche siempre es mayor en los tratamientos con elevados porcentajes, a pesar de no consumirse totalmente la leche suministrada.

c) El coeficiente de correlación lineal entre el incremento de peso y el consumo de L eche nos indica una asociación real entre estas características.

d) La mejor conversión alimenticia láctea se observa en el tratamiento B (5.76); con el suministro inicial del 12 por ciento del peso vivo del ternero. 
e) Los incrementos de peso postdestete obtenidos por los terneros no muestran influencia de la alimentación con leche.

\section{BIBLIOGRAFIA}

AHMED, LA. y K. EL SHAZLY (1978). El destete precoz de los búfalos en Egipto, Revista M undial de Zootecnia, FA O, Roma 12:44-148.

EMPRESA BRASILEIRA DE PESQUISA AGROPECUARIA (EMBRAPA). (1981), Bubalinos: resumos informativos, EM BRAPA, Brasil, $192 \mathrm{p}$.

JOSIFOVICH, J. et al (1990). Destete precoz y engorde de terneros en rodeos de recría, Instituto de Tecnología A gropecuaria (INTA), A rgentina, Inf. № 237, $12 \mathrm{p}$.

LITTLE, T.M. y F.J. HILLS (1981). M étodos estadísticos para la investigación en la A gricultura, Trillas, M éxico, 272 p.

LOPEZ, A. (1989). Nutrición y alimentación de la vaca y el ternero, Albatros, Buenos Aires, $267 \mathrm{p}$.

MARENGO, J. (1983). Estudios Agroclimático en la zona de Jenaro Herrera (Requena-Loreto) y Climática en la Selva Baja Norte del Perú, Tesis U niversidad N acional A graria L a M olina, Lima. 410 p.

ZAM BRAN O, D. et al (1992). Crianza de terneros bubalinos en Selva Baja. En XV Reunión Científica Anual de la Asociación Peruana de Producción A nimal (APPA). Pucallpa, Perú 30 nov-05 dic. 1992. U niversidad Nacional de U cayali, s.p. 
E. Katayama C. y M. Isuiza C.

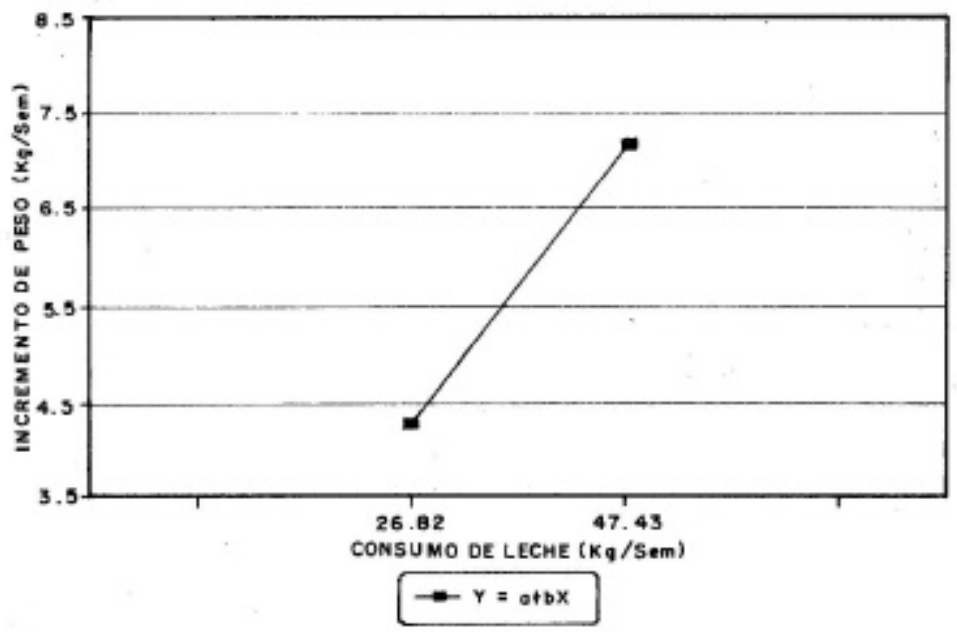

GRAFICO 1 ECUACION Y LINEA DE REGRESION ENTRE CONSUMO DE LECHE E INCREM. DE PESO 